Full length article

\title{
Risk factors for sporadic infections caused by Shiga toxin-producing Escherichia coli: a systematic review and meta-analysis
}

\author{
Jean-Christophe Augustin ${ }^{\mathrm{a}, 1}$, Pauline Kooh ${ }^{\mathrm{b}, *, 1}$, Lapo Mughini-Gras ${ }^{\mathrm{c}, \mathrm{d}}$, Laurent Guillier ${ }^{\mathrm{b}}$, \\ Anne Thébault ${ }^{\mathrm{b}}$, Frédérique Audiat-Perrin ${ }^{\mathrm{b}}$, Vasco Cadavez ${ }^{\mathrm{e}}$, Ursula Gonzales-Barron ${ }^{\mathrm{e}}$, \\ Moez Sanaa ${ }^{\mathrm{b}}$ \\ ${ }^{\text {a }}$ Ecole Nationale Vétérinaire d'Alfort, Maisons-Alfort, France \\ ${ }^{\mathrm{b}}$ Risk Assessment Department, French Agency for Food, Environmental and Occupational Health and Safety (ANSES), Maisons-Alfort, France \\ ${ }^{\mathrm{c}}$ National institute for Public health and the Environment (RIVM), Centre for Infectious Disease Control (CIb), Bilthoven, the Netherlands \\ ${ }^{\mathrm{d}}$ Institute for Risk Assessment Sciences (IRAS), Faculty of Veterinary Medicine, Utrecht University, Utrecht, the Netherlands \\ ${ }^{\mathrm{e}}$ Centro de Investigação de Montanha (CIMO), Instituto Politécnico de Bragança, Campus de Santa Apolónia, Bragança, Portugal
}

\section{A R T I C L E I N F O}

\section{Keywords:}

Research synthesis

observational studies

Shiga toxin-producing E. coli (STEC)

case-control studies

meta-regression

\begin{abstract}
A B S T R A C T
Shiga toxin-producing Escherichia coli (STEC) are an important cause of foodborne disease associated with clinical outcomes ranging from mild intestinal discomfort to haemolytic uremic syndrome, including end-stage renal disease and death. The objective of this study was to synthetize evidence on risk factors for sporadic STEC infection by meta-analysing outcomes from available case-control studies. Suitable scientific articles were identified through a systematic literature search, and assessed for methodological quality. From each study, odds ratios (OR) were extracted along with study characteristics such as the population, design, statistical model used and risk factor hierarchy. Mixed-effects meta-analytical models were adjusted by population type to appropriate data partitions. The quality assessment stage was passed by 29 studies investigating sporadic STEC infection conducted between 1986 and 2013. These studies provided 493 ORs for meta-analysis. The main risk factors for STEC infection were foreign travel, contact with ill people, farm animals or their environment, food consumption and exposure to untreated drinking water. Concerning food exposures, this meta-analysis confirmed known risk factors, such as consumption of beef (especially when undercooked) and other meats (barbecued meat, donner kebab meat and meat casseroles), processed meat, ready-to-eat meat, composite foods, and raw milk consumption by children. Newly identified food vehicles were chicken and fish. Produce (fruits/vegetables) was not associated with sporadic STEC infection.
\end{abstract}

\section{Introduction}

Shiga-toxin-producing Escherichia coli (STEC) are important causative agents of foodborne disease worldwide. STEC infections have been associated with a wide range of clinical outcomes, from mild intestinal discomfort to haemolytic uremic syndrome (HUS) or end-stage renal disease and death (EFSA BIOHAZ Panel, 2013). Young children, immunocompromised persons, and the elderly are at higher risk of developing severe illness. The World Health Organization (WHO) estimated that foodborne STEC caused more than one million illnesses, resulting in more than 100 deaths and nearly 13000 disability-adjusted life years (DALYs) in 2010 (Kirk et al., 2015). In Europe, the incidence of
STEC infection was 1.66 cases per 100,000 population in 2017 (EFSA and ECDC, 2018). The highest incidence of STEC-associated HUS is found in children under 5 years of age (EFSA and ECDC, 2018). Since 2012, STEC epidemiology in Europe has been characterized by a decrease in the incidence of the historically predominant 0157 serogroup, and the increase in that of non-O157 STEC (Bruyand et al., 2019; EFSA and ECDC, 2018)

STEC have been recovered from a wide range of animal species and foods (EFSA BIOHAZ Panel, 2013). The most important reservoirs of this pathogen are ruminants, including cattle, sheep, goats and deer, but STEC have also been isolated from a wide range of domestic and wild mammals, birds and insects; these animals may be less significant

\footnotetext{
* Corresponding author.

E-mail address: Pauline.kooh@anses.fr (P. Kooh).

1 Co-first authors.
} 




Figure 1. PRISMA flow chart of included studies for case-control studies of STEC infection

sources of STEC, but play an important role in their distribution (Gyles, 2007; Mughini-Gras et al., 2018; Persad and LeJeune, 2014). STEC transmission to humans can occur through direct contact with animals, with infected persons and fomites, and through ingestion of food and water (Karch et al., 1999). The relative importance of selected sources and transmission pathways for STEC infections at regional or global levels has been assessed using outbreak data (Greig and Ravel, 2009; Pires et al., 2019), case-control studies of sporadic infections (Devleesschauwer et al., 2019; Kintz et al., 2017), microbial subtyping (Mughini-Gras et al., 2018) and expert elicitations (Hoffmann et al., 2017). These studies have highlighted the importance of beef, produce and dairy in the transmission of the disease.

Two global source attribution studies have been recently published. The first one used data from STEC outbreaks that occurred globally (27 countries between 1998 and 2017) to estimate source attribution from fractions (Pires et al., 2009). The second one is a systematic review (22 case-control studies from 10 countries between 1985 and 2012) and a meta-analysis to determine the association of different food types with sporadic illnesses caused by STEC (Devleesschauwer et al., 2019).

Case-control studies of sporadic disease are a valuable tool to identify risk factors, as well as routes of transmission, food exposures, behavioural and other factors predisposing to disease. Previous metaanalyses of risk factors of sporadic STEC infections have been conducted on a selection of risk factors (Devleesschauwer et al., 2019; Kintz et al., 2017). Herewith, a systematic review and integration of case-control studies were performed to synthesize evidence on the risk factors for sporadic STEC infection (including travel, host-specific factors, and transmission pathways) applying a novel protocol for data categorization scheme and meta-analysis of observational studies proposed by Gonzales-Barron et al. (2019).

\section{Material and methods}

\subsection{Systematic review}

The literature search was conducted in March 2017 using a combination of keywords related to (1) foodborne hazards, "Shiga* Escherichia coli OR Shiga* E coli OR STEC OR VTEC OR EHEC OR O157:H7 OR O26: H11 OR O145:H28 OR O103:H2 OR O111:H8 OR O104:H4"; and (2) study design, "case-control", "observational study", "sporadic"; joined by the logical connector AND. Relevant studies were identified from five bibliographic search engines, Science Direct, PubMed, Scielo, ISI Web of Science and Scopus. No restrictions were defined for the year of the study or type of publication. The search was limited to the languages English, French, Portuguese and Spanish.

Each reference record was screened for relevance for inclusion in the 
Table 1

Characteristics of case-control studies investigating sources of sporadic STEC infections included in the meta-analysis

\begin{tabular}{|c|c|c|c|c|c|c|c|}
\hline Study ID* & Country & Study period & Population & Design & Analysis \& model** & cases/controls & Quality \\
\hline \multirow[t]{2}{*}{ Belongia et al. 2003} & \multirow[t]{2}{*}{ USA } & \multirow[t]{2}{*}{ 1997-1999 } & \multirow[t]{2}{*}{ Children } & \multirow[t]{2}{*}{ Unmatched } & Uni-Chi & 86 O157:H7 & \multirow[t]{2}{*}{ Good } \\
\hline & & & & & Multi-UL & 525 controls & \\
\hline \multirow[t]{2}{*}{ Bielaszewska et al. 1997} & \multirow[t]{2}{*}{ Czech Republic } & 1995 & Children & Unmatched & Uni-Chi & 15 O157/HUS & Good \\
\hline & & & & & & 45 controls & \\
\hline Bryant et al. 1989 & Canada & 1986-1987 & Mixed & Matched & Uni-Chi & 49 O157:H7 & Good \\
\hline & & & & & & 49 controls & \\
\hline & & & & Unmatched & Uni-Chi & 81 O157:H7 & Poor \\
\hline & & & & & & 96 other GI & \\
\hline Byrne et al. 2014 & UK & 2009-2012 & Mixed & Matched & Multi-CL & 67 Non-O157 & Poor \\
\hline & & & & & & 23000157 & \\
\hline Cieslak et al. 1997 & USA & 1993 & Mixed & Matched & Uni-CL & 10 O157:H7 & Good \\
\hline & & & & & & 15 control & \\
\hline Denno et al. 2009 & USA & 2003-2005 & Children & Matched & Uni-CL & 39 O157:H7 & Good \\
\hline & & & & & & 78 controls & \\
\hline Friesema et al. 2015 & Netherlands & 2008-2012 & Children & Unmatched & Uni-UL & 390157 & Good \\
\hline & & & & & Multi-UL & 15 non-O157 & \\
\hline & & & Mixed & & Uni-UL & 910157 & \\
\hline & & & & Unmatched & Multi-UL & 63 non-O157 & \\
\hline & & & & & & 1563 controls & \\
\hline Gianviti et al. 1994 & Italy & 1988-1992 & Children & Matched & Multi-UL & 43 HUS & Good \\
\hline & & & & & & 43 controls & \\
\hline Heusinkveld et al. 2016 & Netherlands & 2013-2014 & Children & Unmatched & Multi-UL & 27 STEC & Good \\
\hline & & & & & & 937 controls & \\
\hline Holton et al. 1999 & Canada & 1991 & Mixed & Matched & Uni-MH & 100 O157:H7 & Good \\
\hline & & & & & Multi-CL & 200 controls & \\
\hline Hundy 2004 & Australia & 2002 & Mixed & Matched & Uni-MH & 11 STEC & Good \\
\hline & & & & & & 22 controls & \\
\hline Jaros et al. 2013 & New Zealand & 2011-2012 & Mixed & Unmatched & Uni-UL & 113 STEC & Good \\
\hline & & & & & Multi-UL & 506 controls & \\
\hline Kassenborg et al. 2004 & USA & 1996-1997 & Mixed & Matched & Uni-CL & 196 O157:H7 & Good \\
\hline & & & Children & & Multi-CL & 372 controls & \\
\hline LeSaux et al. 1993 & Canada & 1990 & Mixed & Matched & Uni-MH & 110 O157:H7 & Good \\
\hline & & & & & & 220 controls & \\
\hline Locking et al. 2001 & UK & 1996-1999 & Mixed & Matched & Uni-CL & 183 O157/HUS & Good \\
\hline & & & & & Multi-CL & 545 controls & \\
\hline MacDonald et al. 1988 & USA & $1985-1986$ & Mixed & Matched & Uni-MH & 24 O157:H7 & Good \\
\hline & & & & & & 48 controls & \\
\hline McPherson et al. 2009 & Australia & 2003-2007 & Mixed & Unmatched & Uni-Chi & 430157 & Good \\
\hline & & & & & Multi-UL & 71 non-O157 & \\
\hline & & & & & & 304 controls & \\
\hline Mead et al. 1997 & USA & 1994 & Mixed & Matched & Uni-MH,CL & 22 O157:H7 & Good \\
\hline & & & & & & 44 controls & \\
\hline O'Brien et al. 2001 & UK & 1996-1997 & Mixed & Unmatched & Multi-UL & 3690157 & Good \\
\hline & & & & & & 511 controls & \\
\hline Parry et al. 1998 & UK & 1994-1996 & Mixed & Matched & Uni-MH & 850157 & Good \\
\hline & & & & & Multi-CL & 142 controls & \\
\hline Pierard et al. 1999 & Belgium & 1998 & Children & Matched & Uni-MH & 290157 & Good \\
\hline & & & & & & 55 controls & \\
\hline & & & Mixed & Matched & Uni-MH & 21 eaeA-pos & \\
\hline & & & & & & 40 controls & \\
\hline & & & Mixed & Matched & Uni-MH & 16 eaeA-pos & \\
\hline & & & & & & 29 controls & \\
\hline & & & Mixed & Matched & Multi-CL & 37 STEC & \\
\hline & & & & & & 69 controls & \\
\hline Rivas et al. 2008 & Argentina & 2001-2002 & Children & Matched & Uni-CL & 150 STEC & Poor \\
\hline & & & & & Multi-CL & 299 controls & \\
\hline & & & & & Uni-Chi & 580157 & \\
\hline & & & & & & 116 controls & \\
\hline & & & & & Uni-Chi & 38 non-O157 & \\
\hline & & & & & & 75 controls & \\
\hline Rivero et al. 2011 & Argentina & 2010 & Children & Unmatched & Uni-Chi & 62 STEC/HUS & Poor \\
\hline & & & & & Multi-UL & 372 other GI & \\
\hline Rowe et al. 1993 & Canada & 1990 & Children & Unmatched & Uni-Chi & 34 HUS & Good \\
\hline & & & & & & 102 controls & \\
\hline Slutsker et al.1998 & USA & 1990-1992 & Mixed & Matched & Uni-CL & 73 O157:H7 & Good \\
\hline & & & & & & 142 controls & \\
\hline Vaillant et al. 2009 & France & $2000-2001$ & Children & Matched & Uni-MH & 105 HUS & Good \\
\hline & & & & & Multi-CL & 196 controls & \\
\hline Voetsch et al. 2007 & USA & $1999-2000$ & Mixed & Matched & Multi-UL/CL & 179 STEC adults & Good \\
\hline & & & Children & & & 104 STEC child & \\
\hline & & & & & & 534 controls & \\
\hline Wang et al. 2013 & Canada & $2009-2011$ & Mixed & Unmatched & Uni-Chi & 63 non-O157 & Poor \\
\hline & & & & & & 154 O157/HUS & \\
\hline Werber et al. 2007 & Germany & 2001-2003 & Children & Matched & Uni-MH & 145 STEC child & Good \\
\hline
\end{tabular}


Table 1 (continued)

\begin{tabular}{|c|c|c|c|c|c|c|c|}
\hline Study ID* & Country & Study period & Population & Design & Analysis \& model ${ }^{* *}$ & cases/controls & Quality \\
\hline & & & Mixed & Matched & $\begin{array}{l}\text { Multi-CL } \\
\text { Uni-MH } \\
\text { Multi-CL }\end{array}$ & $\begin{array}{l}145 \text { controls } \\
57 \text { STEC adults } \\
57 \text { controls }\end{array}$ & \\
\hline
\end{tabular}


conditional logistic (UL) or conditional logistic (CL)

meta-analysis study. The methodological quality of the candidate studies were assessed using pre-defined quality criteria comprising (1) appropriate selection of the controls, (2) adjustment to correct for confounders, (3) comparability between cases and controls, (4) acceptable responses rates for the exposed and control groups, (5) data analysis appropriate to the study design, (6) provision of odds ratio (OR) with confidence interval or p-value, or provision of sufficient data to calculate ORs, (7) overall quality of the study (Gonzales-Barron et al., 2019). Primary studies that passed the screening for relevance were marked as having potential for bias if they failed to meet at least one of the methodological quality assessment criteria.

Data from primary studies were then extracted using a standardised spreadsheet. Data extracted included the relevant study characteristics (location, time period, population, serotype/phage type, case definition, design, sample size of the groups, type of model, etc.), the categorized risk factors, the setting, the handling practices and the outcome of the study (Odds Ratio [OR]).

A data categorisation scheme was established to hierarchically group the risk factors into travel, host-specific factors and pathways of exposure (i.e., person-to-person, animal, environment and food routes) (Gonzales-Barron et al., 2019). The variable "population" was stratified into mixed and children. The child population was further subdivided into young (younger than 6 years) and older children (older than 6 years).

\subsection{Data synthesis}

As described in Gonzales-Barron et al. (2019), the joint meta-analytical data was first described using basic statistics. Next, data were partitioned into subsets of categories of risk factors. Meta-analytical models were then fitted to each of the data partitions or subsets in order to estimate the overall OR due to-travel, host specific factors and transmission pathways related to person-to-person contagion, animal contact, environmental exposures and food vehicles. The meta-analytical models were fitted separately for each population type. For some food classes, the effects of handling (i.e., eating raw, undercooked) and setting (i.e., eating food prepared outside the home) on the overall OR were assessed by calculating the ratio of the mean OR when food was mishandled (or, alternatively, when food was prepared outside the home) to the base OR.

The statistical analysis was designed to assess the effect of the geographical region, the study period and the type of analysis (univariate/multivariate) on the final result. The objective of the region-specific meta-analysis was to inform the decision on the geographical regions that should be maintained for the subsequent pooling of ORs. A geographical region (Asia, North America, South America, Africa, Europe, Oceania) was removed from a particular meta-analysis partition only if its pooled ORs were different from those associated with the other regions, or if less than 3 ORs represented the region (Gonzales-Barron et al., 2019)

All meta-analytical models were weighted random-effects linear regression models. Once a meta- analytical model was fitted, influential diagnostics statistics were applied in order to remove any influential observation originating from studies marked as having potential for bias. Publication bias was assessed by funnel plots and statistical tests investigating the effect of the study sample size on the ORs (Tables 2, 3 and 4) (Gonzales-Barron et al., 2019). Heterogeneity between studies was assessed by different indicators, such as the between-study variability $\left(\tau^{2}\right)$, the QE test investigating residual heterogeneity, the variance of residuals and the intra-class correlation $\mathrm{I}^{2}$ (Gonzales-Barron et al., 2019). Publication bias and remaining heterogeneity were not further corrected for, but were taken into account for the interpretation of the results.

All analyses were produced in the R software (R Development Core Team, 2008) implemented with the metafor package (Viechtbauer, 2010).

\section{Results}

\subsection{Descriptive statistics}

From the 4718 references initially identified, a total of 253 articles passed the relevance screening, and finally 29 case-control studies passed the quality assessment stage (Fig. 1). Table 1 summarizes the main features of the case-control studies included in this meta-analysis. These 29 studies were conducted between 1986 and 2013 and jointly provided 493 ORs for meta-analysis. A total of 213 ORs were retrieved from 17 case-control studies performed before the year 2000, while 280 ORs were obtained from 12 case-control studies performed after 2000. The majority of primary studies investigated STEC infection or HUS caused by 0157:H7 strains (12 studies), 0157 strains (7 studies), nonO157 strains (5 studies) and undifferentiated strains (9 studies), producing $20 \%, 30 \%, 12 \%$, and $38 \%$ of the ORs, respectively. In 26 primary studies, the STEC infections were laboratory-confirmed, while in three studies (Cieslak et al., 1997; Gianviti et al., 1994; Locking et al., 2001), there was no indication that all of the cases were confirmed. The countries whose case-control studies presented the largest body of results for sporadic STEC infection were Argentina (86 ORs), USA (77 ORs), UK (76 ORs), Australia (59 ORs), Germany (57 ORs) and Canada (43 ORs), which produced altogether $\sim 80 \%$ of the ORs retrieved.

Fourteen studies investigated pathways of exposure in children and 20 in the mixed population; however, these case-control studies did not add up to the total number of 29 , because five of them presented separate results for the mixed and the child population (Friesema et al., 2015; Kassenborg et al., 2004; Piérard et al., 1999; Voetsch et al., 2007; Werber et al., 2007). Whereas 297 ORs (61\% of the data) originated from exposures evaluated in the mixed population, 196 ORs (39\%) were quantified from ill children. The "children" category was further broken down into two subcategories: "young children" (70 ORs), comprising infants and children up to 6 years old; and "older children" (126 ORs), comprising children up to 16 years of age. It is important to remember that the child population categories are not mutually exclusive. As a rule, because of their distinct routes of exposure, the ORs for children and mixed populations were not combined in a single meta-analysis model, but were analyzed in separate meta-analyses. Data from both age groups were merged only when the ORs belonging to the child population were too few to run a separate meta-analysis model.

With regards to the risk factor classes, sporadic illness investigations focused much more on the multiple pathways of transmission (477 ORs) than on host-specific factors (7 ORs) or travel (9 ORs). Most of the routes of transmission for STEC infection identified in the systematic review concerned exposures about food consumption, for which a total of 282 ORs were extracted.

During methodological quality assessment, potential for selection 
Table 2

Results of the meta-analysis on main risk factors for sporadic STEC infection

\begin{tabular}{|c|c|c|c|c|c|c|c|c|}
\hline Population & Geographical area & Risk factor & $\begin{array}{l}\text { Pooled OR } \\
{[95 \% \mathrm{CI}]}\end{array}$ & $\mathrm{N} / \mathrm{n}^{*}$ & $\begin{array}{l}\text { p-value risk } \\
\text { factor }\end{array}$ & $\begin{array}{l}\text { Publication bias } \\
\text { p-value }\end{array}$ & $\begin{array}{l}\text { Points } \\
\text { removed }^{* *}\end{array}$ & Heterogeneity analysis*** \\
\hline \multicolumn{9}{|l|}{ Travel } \\
\hline \multirow[t]{2}{*}{ Mixed } & \multirow[t]{2}{*}{ All } & Any & $\begin{array}{l}2.398[1.690 \\
-3.400]\end{array}$ & $2 / 3$ & $<.0001$ & \multirow[t]{2}{*}{0.011} & \multirow[t]{2}{*}{0} & $\begin{array}{l}\tau^{2}=0.000 \\
Q E(d f=6)=15.49, p- \\
\text { val }=0.017\end{array}$ \\
\hline & & Abroad & $\begin{array}{l}5.496[3.087 \\
-9.784]\end{array}$ & $2 / 3$ & $<.0001$ & & & $\begin{array}{l}\mathrm{S}^{2}=0.840 \\
\mathrm{I}^{2}=0.0\end{array}$ \\
\hline \multicolumn{9}{|l|}{ Host specific } \\
\hline Mixed & All & Antibiotics & $\begin{array}{l}1.768[1.086 \\
-2.878]\end{array}$ & $2 / 5$ & 0.022 & 0.065 & 0 & $\begin{array}{l}\tau^{2}=0.023 \\
Q E(d f=5)=5.244, p- \\
\text { val }=0.387 \\
\mathrm{~S}^{2}=0.231 \\
\mathrm{I}^{2}=11.18\end{array}$ \\
\hline \multicolumn{9}{|l|}{ Animals } \\
\hline \multirow[t]{2}{*}{ Mixed } & \multirow[t]{2}{*}{ No data from USA } & $\begin{array}{l}\text { Occupational } \\
\text { exposure }\end{array}$ & $\begin{array}{l}2.940[2.062 \\
-4.192]\end{array}$ & $4 / 10$ & $<.0001$ & \multirow[t]{2}{*}{0.134} & \multirow[t]{2}{*}{1} & $\begin{array}{l}\tau^{2}=0.257 \\
\mathrm{QE}(\mathrm{df}=28)=55.48, \mathrm{p}- \\
\mathrm{val}=0.002\end{array}$ \\
\hline & & Farm animals & $\begin{array}{l}1.936[1.260 \\
-2.973]\end{array}$ & $7 / 18$ & 0.003 & & & $\begin{array}{l}\mathrm{S}^{2}=0.339 \\
\mathrm{I}^{2}=43.04\end{array}$ \\
\hline Young Children & All & Farm animals & $\begin{array}{l}4.357[2.471 \\
-7.683]\end{array}$ & $2 / 4$ & $<.0001$ & 0.264 & 0 & $\begin{array}{l}\tau^{2}=0.000 \\
Q E(d f=6)=3.288, p- \\
\text { val }=0.772 \\
S^{2}=0.120 \\
I^{2}=0.0\end{array}$ \\
\hline \multirow[t]{2}{*}{ Older children } & All & Before 2000 & $\begin{array}{l}1.850[1.516 \\
-2.257]\end{array}$ & $3 / 10$ & $<.0001$ & 0.882 & 0 & $\begin{array}{l}\tau^{2}=0.000 \\
Q E(d f=21)=11.545, p- \\
\text { val }=0.951 \\
S^{2}=0.157\end{array}$ \\
\hline & & After 2000 & $\begin{array}{l}3.247[2.375 \\
-4.440]\end{array}$ & $3 / 13$ & $<.0001$ & & & $\mathrm{I}^{2}=0.0$ \\
\hline Environment & & & & & & & & \\
\hline Mixed (at) & All & Farm environment & $\begin{array}{l}2.972[2.234 \\
-3.953]\end{array}$ & $8 / 25$ & $<.0001$ & 0.480 & 0 & $\begin{array}{l}\tau^{2}=0.223 \\
Q E(d f=51)=72.78, p- \\
\text { val }=0.024\end{array}$ \\
\hline & & $\begin{array}{l}\text { Untreated } \\
\text { drinking water }\end{array}$ & $\begin{array}{l}2.551[1.681 \\
-3.872]\end{array}$ & $6 / 13$ & 0.001 & & & $\begin{array}{l}\mathrm{S}^{2}=0.323 \\
\mathrm{I}^{2}=40.78\end{array}$ \\
\hline & & $\begin{array}{l}\text { Recreational } \\
\text { water }\end{array}$ & $\begin{array}{l}1.477[1.040 \\
-2.098]\end{array}$ & $7 / 13$ & 0.060 & & & \\
\hline & & Playground & $\begin{array}{l}2.676[1.487 \\
-4.817]\end{array}$ & $2 / 5$ & 0.001 & & & \\
\hline Young Children & $\begin{array}{l}\text { South America } \\
\text { removed }\end{array}$ & Farm environment & $\begin{array}{l}5.346[2.516 \\
-11.36]\end{array}$ & $2 / 3$ & $<.0001$ & 0.857 & 0 & $\begin{array}{l}\tau^{2}=0.000 \\
Q E(d f=8)=4.247, p^{-} \\
\text {val }=0.834 \\
S^{2}=0.245 \\
I^{2}=0.0\end{array}$ \\
\hline Older Children & $\begin{array}{l}\text { South America } \\
\text { removed }\end{array}$ & Farm environment & $\begin{array}{l}1.689[1.350 \\
-2.113]\end{array}$ & $2 / 5$ & $<.0001$ & 0.187 & 0 & $\begin{array}{l}\tau^{2}=0.216 \\
\mathrm{QE}(\mathrm{df}=10)=24.78, \mathrm{p}- \\
\mathrm{val}=0.006 \\
\mathrm{~S}^{2}=0.531\end{array}$ \\
\hline & & $\begin{array}{l}\text { Untreated } \\
\text { drinking water }\end{array}$ & $\begin{array}{l}2.505[1.298 \\
-4.835]\end{array}$ & $4 / 5$ & 0.006 & & & $\mathrm{I}^{2}=28.94$ \\
\hline Person to person & & & & & & & & \\
\hline Mixed & $\begin{array}{l}\text { South America } \\
\text { removed }\end{array}$ & & $\begin{array}{l}2.548[1.283 \\
-5.060]\end{array}$ & $7 / 11$ & 0.008 & 0.101 & 0 & $\tau^{2}=0.744$ \\
\hline Young Children & & & $\begin{array}{l}2.503[1.218 \\
-5.147]\end{array}$ & $2 / 3$ & 0.013 & & & $\begin{array}{l}\mathrm{QE}(\mathrm{df}=18)=37.924, \mathrm{p}- \\
\mathrm{val}=0.004 \\
\mathrm{~S}^{2}=0.697\end{array}$ \\
\hline Older Children & & & $\begin{array}{l}5.722[3.855 \\
-8.494]\end{array}$ & $3 / 7$ & $<.0001$ & & & $\mathrm{I}^{2}=51.637$ \\
\hline $\begin{array}{l}\text { Food } \\
\text { Mixed }\end{array}$ & All & Meat & $\begin{array}{l}1.809[1.397 \\
-2.342]\end{array}$ & $\begin{array}{l}18 / \\
119\end{array}$ & $<.0001$ & $<.0001$ & 3 & $\begin{array}{l}\tau^{2}=1.061 \\
\mathrm{QE}(\mathrm{df}=164)=361.9, \mathrm{p}-\mathrm{val}< \\
.0001\end{array}$ \\
\hline & & Composite & $\begin{array}{l}1.587[1.085 \\
-2.322]\end{array}$ & $7 / 15$ & 0.017 & & & $\mathrm{~S}^{2}=0.590$ \\
\hline & & Seafood & $\begin{array}{l}1.720[1.100 \\
-2.687]\end{array}$ & $3 / 5$ & 0.017 & & & $\mathrm{I}^{2}=64.26$ \\
\hline Young Children & All & Meat & $\begin{array}{l}1.688[1.103 \\
-2.583]\end{array}$ & $3 / 20$ & 0.016 & 0.855 & 2 & $\begin{array}{l}\tau^{2}=0.796 \\
Q E(d f=33)=38.40, p- \\
\text { val }=0.238 \\
S^{2}=0.632 \\
I^{2}=55.74\end{array}$ \\
\hline
\end{tabular}


Table 2 (continued)

\begin{tabular}{|c|c|c|c|c|c|c|c|c|}
\hline Population & Geographical area & Risk factor & $\begin{array}{l}\text { Pooled OR } \\
{[95 \% \mathrm{CI}]}\end{array}$ & $\mathrm{N} / \mathrm{n}^{*}$ & $\begin{array}{l}\text { p-value risk } \\
\text { factor }\end{array}$ & $\begin{array}{l}\text { Publication bias } \\
\text { p-value }\end{array}$ & $\begin{array}{l}\text { Points } \\
\text { removed** }\end{array}$ & Heterogeneity analysis*** \\
\hline \multirow[t]{2}{*}{ Older children } & \multirow[t]{2}{*}{ All } & Meat & $\begin{array}{l}1.888[1.364 \\
-2.613]\end{array}$ & $6 / 48$ & $<.0001$ & 0.135 & 0 & $\begin{array}{l}\tau^{2}=0.641 \\
\mathrm{QE}(\mathrm{df}=59)=116.8, \mathrm{p}-\mathrm{val}< \\
.0001 \\
\mathrm{~S}^{2}=0.753\end{array}$ \\
\hline & & Composite & $\begin{array}{l}3.118[1.555 \\
-6.250]\end{array}$ & $2 / 9$ & 0.001 & & & $\mathrm{I}^{2}=45.98$ \\
\hline \multirow[t]{3}{*}{$\begin{array}{l}\text { Children (both } \\
\text { age groups) }\end{array}$} & \multirow[t]{3}{*}{ All } & Dairy & $\begin{array}{l}2.240[1.161 \\
-4.320]\end{array}$ & $7 / 13$ & 0.016 & 0.165 & 2 & $\tau^{2}=0.970$ \\
\hline & & Meat & $\begin{array}{l}1.829 \text { [1.407- } \\
2.379]\end{array}$ & $8 / 69$ & $<.0001$ & & & \multirow{2}{*}{$\begin{array}{l}\mathrm{QE}(\mathrm{df}=96)=152.3, \mathrm{p}- \\
\mathrm{val}=0.0002, \mathrm{~S}^{2}=0.724 \\
\mathrm{I}^{2}=57.24\end{array}$} \\
\hline & & Composite & $\begin{array}{l}3.556[2.202 \\
-5.742]\end{array}$ & $3 / 10$ & $<.0001$ & & & \\
\hline
\end{tabular}



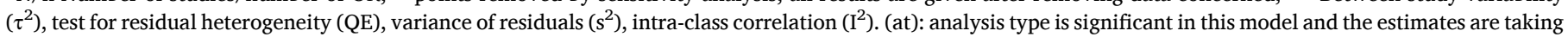
this effect into account.

Table 3

Results of the meta-analysis on disaggregated risk factors for sporadic STEC infection

\begin{tabular}{|c|c|c|c|c|c|c|c|c|}
\hline Risk Factor & Popula-tion & $\begin{array}{l}\text { Risk factor } \\
\text { Precise }\end{array}$ & $\begin{array}{l}\text { Pooled OR } \\
{[95 \% \mathrm{CI}]}\end{array}$ & $\mathrm{N} / \mathrm{n}^{*}$ & $\begin{array}{l}\mathrm{p} \text {-value of risk } \\
\text { factor }\end{array}$ & $\begin{array}{l}\text { Publica-tion bias } \\
\text { p-value }\end{array}$ & $\begin{array}{l}\text { Points remo- } \\
\text { ved }^{* *}\end{array}$ & Heterogeneity analysis ${ }^{* * *}$ \\
\hline \multirow[t]{4}{*}{ Meat } & \multirow[t]{4}{*}{ Mixed (all regions) } & Beef & $\begin{array}{l}1.904[1.462- \\
2.479]\end{array}$ & $\begin{array}{l}14 / \\
57\end{array}$ & $<.0001$ & \multirow[t]{4}{*}{$<.0001$} & \multirow[t]{4}{*}{0} & \multirow{4}{*}{$\begin{array}{l}\tau^{2}=0.848 \\
Q E(d f=113)=218.9, p- \\
\text { val }<.0001 \\
S^{2}=0.562 \\
I^{2}=60.16\end{array}$} \\
\hline & & Poultry & $\begin{array}{l}3.549[1.990- \\
6.330]\end{array}$ & $4 / 7$ & $<.0001$ & & & \\
\hline & & $\begin{array}{l}\text { Processed } \\
\text { meat }\end{array}$ & $\begin{array}{l}1.445[1.066- \\
1.959]\end{array}$ & $8 / 28$ & 0.018 & & & \\
\hline & & Others & $\begin{array}{l}1.803[1.389- \\
2.340]\end{array}$ & $8 / 20$ & $<.0001$ & & & \\
\hline \multirow[t]{2}{*}{ Meat } & \multirow[t]{2}{*}{$\begin{array}{l}\text { Young Children (all } \\
\text { regions) }\end{array}$} & Others & $\begin{array}{l}2.574[1.441- \\
4.598]\end{array}$ & $2 / 5$ & 0.001 & \multirow[t]{2}{*}{0.798} & \multirow[t]{2}{*}{0} & \multirow{7}{*}{$\begin{array}{l}\tau^{2}=0.000 \\
\mathrm{QE}(\mathrm{df}=17)=6.775, \mathrm{p} \text {-val } \\
=0.986 \\
\mathrm{~S}^{2}=0.126 \\
\mathrm{I}^{2}=0.000 \\
\tau^{2}=0.317 \\
\mathrm{QE}(\mathrm{df}=45)=78.21, \mathrm{p} \text {-val } \\
=0.002 \\
\mathrm{~S}^{2}=0.642 \\
\mathrm{I}^{2}=33.01 \\
\tau^{2}=0.187 \\
\mathrm{QE}(\mathrm{df}=64)=94.48, \mathrm{p} \text {-val } \\
=0.008 \\
\mathrm{~S}^{2}=0.592 \\
\mathrm{I}^{2}=23.99\end{array}$} \\
\hline & & $\begin{array}{l}\text { Processed } \\
\text { meat }\end{array}$ & $\begin{array}{l}1.830[1.093- \\
3.065]\end{array}$ & $2 / 5$ & 0.022 & & & \\
\hline \multirow[t]{2}{*}{ Meat } & \multirow[t]{2}{*}{ Older children (all regions) } & Others & $\begin{array}{l}2.246[1.344- \\
3.753]\end{array}$ & $3 / 6$ & 0.002 & \multirow[t]{2}{*}{0.680} & \multirow[t]{2}{*}{0} & \\
\hline & & Beef & $\begin{array}{l}2.216[1.399- \\
3.509]\end{array}$ & $4 / 36$ & 0.001 & & & \\
\hline \multirow[t]{3}{*}{ Meat } & \multirow[t]{3}{*}{$\begin{array}{l}\text { Children (both age groups) } \\
\text { (all regions) }\end{array}$} & Others & $\begin{array}{l}2.413[1.643- \\
3.544]\end{array}$ & $5 / 11$ & $<.0001$ & \multirow[t]{3}{*}{0.848} & \multirow[t]{3}{*}{1} & \\
\hline & & Beef & $\begin{array}{l}1.846[1.260- \\
2.704]\end{array}$ & $6 / 43$ & 0.002 & & & \\
\hline & & $\begin{array}{l}\text { Processed } \\
\text { meat }\end{array}$ & $\begin{array}{l}1.810[1.322- \\
2.480]\end{array}$ & $3 / 10$ & 0.0002 & & & \\
\hline Beef & All populations & & $\begin{array}{l}1.877[1.513- \\
2.329]\end{array}$ & $\begin{array}{l}17 / \\
100\end{array}$ & $<.0001$ & 0.054 & 0 & $\begin{array}{l}\tau^{2}=0.132 \\
\mathrm{Q}(\mathrm{df}=99)=241.3, \mathrm{p} \text {-val } \\
<.0001 \\
\mathrm{~S}^{2}=0.772 \\
\mathrm{I}^{2}=14.58\end{array}$ \\
\hline $\begin{array}{l}\text { Processed } \\
\text { meat }\end{array}$ & All populations & & $\begin{array}{l}1.513[1.227- \\
1.866]\end{array}$ & $\begin{array}{l}10 / \\
39\end{array}$ & 0.0001 & 0.296 & 0 & $\begin{array}{l}\tau^{2}=0.069 \\
Q(d f=38)=52.05 \text { p-val } \\
=0.064 \\
\mathrm{~S}^{2}=0.370 \\
\mathrm{I}^{2}=15.66\end{array}$ \\
\hline Dairy & Young Children & Raw Milk & $\begin{array}{l}7.188[2.387- \\
21.64]\end{array}$ & $2 / 3$ & 0.0005 & 0.660 & 1 & $\begin{array}{l}\tau^{2}=0.000 \\
Q E(d f=6)=6.185, p-v a l \\
=0.403 \\
S^{2}=0.886 \\
I^{2}=0.000\end{array}$ \\
\hline Raw milk & $\begin{array}{l}\text { Children (both age groups) } \\
\text { (all regions) }\end{array}$ & & $\begin{array}{l}4.117[1.043- \\
16.25]\end{array}$ & $4 / 5$ & 0.043 & $<.0001$ & 0 & $\begin{array}{l}\tau^{2}=1.240 \\
Q(d f=4)=15.86, p-v a l= \\
0.003 \\
\mathrm{~S}^{2}=2.147 \\
\mathrm{I}^{2}=36.62\end{array}$ \\
\hline Composite & Mixed (all regions) & Fast food & $\begin{array}{l}1.809[1.011- \\
3.238]\end{array}$ & $3 / 3$ & 0.0001 & 0.001 & 0 & $\begin{array}{l}\tau^{2}=0.463 \\
\mathrm{QE}(\mathrm{df}=13)=48.83, \mathrm{p}-\mathrm{val} \\
<.0001 \\
\mathrm{~S}^{2}=0.382 \\
\mathrm{I}^{2}=54.79\end{array}$ \\
\hline RTE & Mixed (all regions) & Meat & $\begin{array}{l}1.387[1.090- \\
1.766]\end{array}$ & $8 / 24$ & 0.008 & 0.634 & 1 & $\begin{array}{l}\tau^{2}=0,152 \\
\mathrm{QE}(\mathrm{df}=26)=41.76, \mathrm{p}-\mathrm{val} \\
=0.026\end{array}$ \\
\hline
\end{tabular}

(continued on next page) 
Table 3 (continued)

\begin{tabular}{|c|c|c|c|c|c|c|c|c|}
\hline Risk Factor & Popula-tion & $\begin{array}{l}\text { Risk factor } \\
\text { Precise }\end{array}$ & $\begin{array}{l}\text { Pooled OR } \\
{[95 \% \mathrm{CI}]}\end{array}$ & $\mathrm{N} / \mathrm{n}^{*}$ & $\begin{array}{l}\mathrm{p} \text {-value of risk } \\
\text { factor }\end{array}$ & $\begin{array}{l}\text { Publica-tion bias } \\
\text { p-value }\end{array}$ & $\begin{array}{l}\text { Points remo- } \\
\text { ved }^{* *}\end{array}$ & Heterogeneity analysis ${ }^{* * *}$ \\
\hline & & & & & & & & $\begin{array}{l}\mathrm{S}^{2}=0.417 \\
\mathrm{I}^{2}=26.72\end{array}$ \\
\hline RTE meat & $\begin{array}{l}\text { Children (both age groups) } \\
\text { (all regions) }\end{array}$ & & $\begin{array}{l}1.951[1.424- \\
2.672]\end{array}$ & $3 / 9$ & $<.0001$ & 0.247 & 0 & $\begin{array}{l}\tau^{2}=0.000 \\
Q(d f=8)=15.35, \mathrm{p}-\mathrm{val}= \\
0.052 \\
\mathrm{~S}^{2}=0.699 \\
\mathrm{I}^{2}=0.000\end{array}$ \\
\hline BBQ meat & & & $\begin{array}{l}1.516[1.000- \\
2.298]\end{array}$ & $2 / 3$ & 0.0498 & 0.559 & 0 & $\begin{array}{l}\tau^{2}=0.000 \\
\mathrm{Q}(\mathrm{df}=2)=2.375, \mathrm{p}-\mathrm{val}= \\
0.305 \\
\mathrm{~S}^{2}=0.229 \\
\mathrm{I}^{2}=0.000\end{array}$ \\
\hline
\end{tabular}

${ }^{*} \mathrm{~N} / \mathrm{n}$ Number of studies/number of OR;

** points removed by sensitivity analysis, all results are given after removing data concerned;

B*:* Between-study variability $\left(\tau^{2}\right)$, test for residual heterogeneity $(\mathrm{QE})$, variance of residuals $\left(\mathrm{s}^{2}\right)$, intra-class correlation $\left(\mathrm{I}^{2}\right)$.

bias status was assigned to four case-control studies since, in those, the controls were not healthy individuals but people who had diarrhoea caused by other gastrointestinal infections (Bryant et al., 1989; Rivero et al., 2011), STEC O157 infections (Byrne et al., 2014; Wang et al., 2013). As it is not clear whether these controls shared routes of exposure with the case patients, the ORs extracted from these studies were marked as having potential for bias. Finally, the ORs from Rivas et al. (2008) were assigned the potential-for-bias status because although they used a matched design, from the data presented in the article, only crude ORs could be calculated. These five case-control studies provided 69 potentially-biased ORs whose influence on the meta-analysed OR estimates was appraised by means of Cook's distance. Nineteen case-control studies employed a matched experimental design and produced a total of 320 categorized ORs (Table 1). Bringing together the matched and unmatched designs, 359 of the extracted ORs were not adjusted by any confounder (crude ORs), while only 134 ORs were adjusted using either Mantel-Haenzel or logistic regressions.

\subsection{Meta-analysis}

For every data partition, the meta-analysed risk factors are presented in summary tables only when significant (Tables 2 and 3). Pooled ORs were considered significant when the lower bound of the $95 \%$ CI was equal or greater than 1 . Non-significant results on main risk factors are presented in the Supplementary Material 2. More detailed descriptive results, in particular funnel plots, forest plots, are in a complete report available upon request.

\subsubsection{Meta-analysis for travel and host-specific risk factors}

The meta-analysis showed that domestic and foreign travel increased the risk of STEC infection. On average, people who travelled had 2.398 (95\% CI: 1.690-3.400) greater odds of acquiring STEC infection than people who did not travel at all, and international travelling was the most important factor (pooled OR=5.496; 95\% CI: 3.087-9.784). The meta-analysis on host-specific factors revealed that, on average, people who used antibiotics had a 1.768-fold (95\% CI: 1.086-2.878) greater risk of acquiring STEC infection than those who did not take such medication.

\subsubsection{Meta-analysis for animal-, environment-, and person-to-person-} related pathways of transmission

For the mixed and children populations, contact with farm animals and occupational exposure, i.e. working with livestock or in meat processing establishments, appeared to be an important source of STEC infection (pooled OR ranging from 1.850 to 4.357, Table 2).

The general meta-analyses showed the relevance of the environmental pathways as a whole in the transmission of STEC in the three population classes. In the meta-analysis stratified by environmental pathway, the mixed population in daily contact with farm environments, those drinking untreated water or in contact with recreational water or soil were at increased risk of STEC infection (pooled OR ranging from 1.477 to 2.972 , Table 2). Alike the mixed population, in young children, contact with the farm environment was a significant risk factor for STEC transmission (pooled $\mathrm{OR}=5.346$; $95 \%$ CI: 2.516-11.361, Table 2). The meta-analyses on animal contact pathways showed that case-control

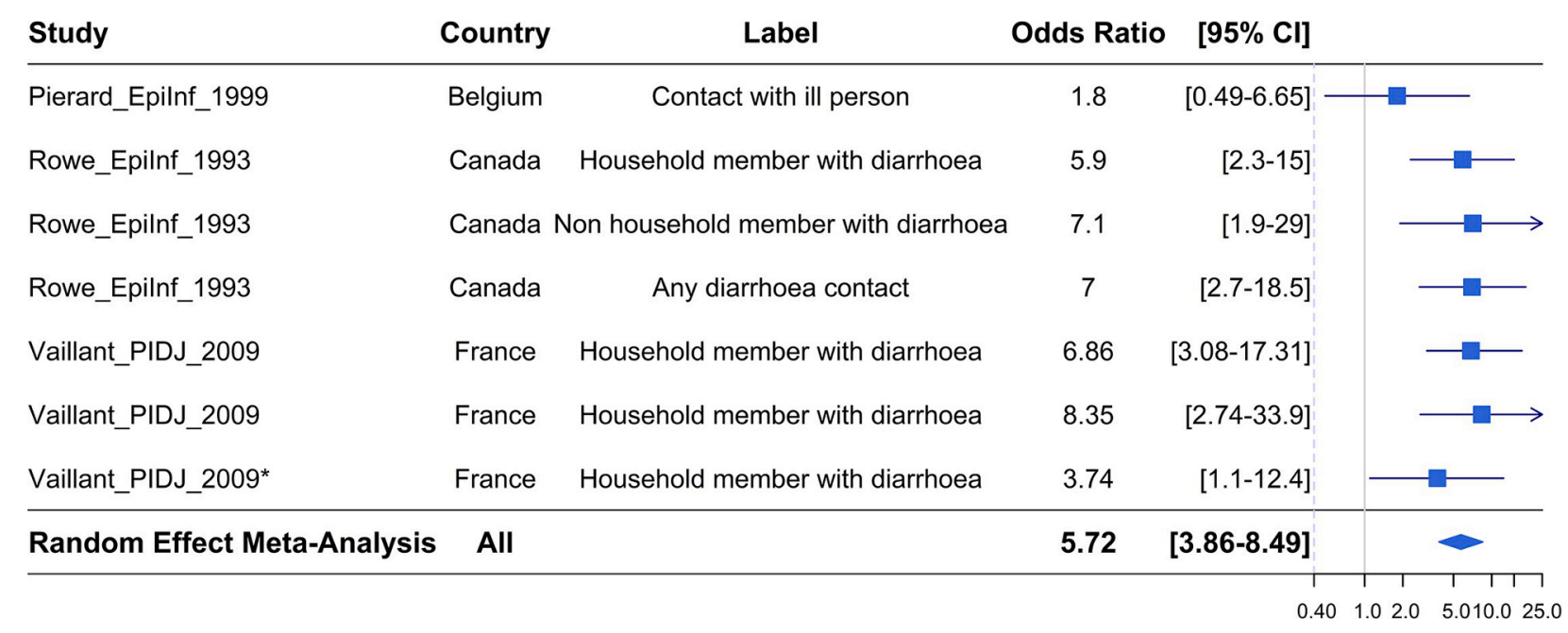

Figure 2. Forest-plot of the association of STEC infection with person-to-person transmission in old children (n=7) (* adjusted OR) 
Table 4

Effect of food handling on the pooled OR of risk factors associated with sporadic STEC infection

\begin{tabular}{|c|c|c|c|c|c|c|c|c|}
\hline $\begin{array}{l}\text { Risk } \\
\text { Factor }\end{array}$ & Risk factor & $\begin{array}{l}\text { Pooled OR }[95 \% \\
\text { CI] }\end{array}$ & $\mathrm{N} / \mathrm{n}^{*}$ & $\begin{array}{l}\text { p-value of risk } \\
\text { factor }\end{array}$ & $\begin{array}{l}\text { Increase ratio in OR } \\
{[95 \% \mathrm{CI}]}\end{array}$ & $\begin{array}{l}\text { Points } \\
\text { removed }\end{array}$ & $\begin{array}{l}\text { Publication bias p- } \\
\text { value }\end{array}$ & Heterogeneity analysis ${ }^{* * *}$ \\
\hline \multirow[t]{3}{*}{ Beef (at) } & Undercooked & $\begin{array}{l}3.240 \text { [1.930 - } \\
5.441]\end{array}$ & $14 / 54$ & $<.0001$ & $1.470[1.217-1.776]$ & 0 & 0.005 & \multirow{3}{*}{$\begin{array}{l}\tau^{2}=0.099 \\
\mathrm{QE}(\mathrm{df}=127)=228.8, \mathrm{p} \text {-val } \\
<.0001 \\
\mathrm{~S}^{2}=0.628 \\
\mathrm{I}^{2}=13.57\end{array}$} \\
\hline & Eating out & $\begin{array}{l}2.438[1.419- \\
4.191]\end{array}$ & $12 / 34$ & 0.350 & $1.106[0.895-1.368]$ & & & \\
\hline & Base (beef) & $\begin{array}{l}2.204[1.585- \\
3.064]\end{array}$ & $\begin{array}{l}18 / 97 \\
\text { (s) } \\
17 / 77 \\
\text { (u) }\end{array}$ & $<.0001$ & - & & & \\
\hline \multirow[t]{2}{*}{ Milk } & Raw & $\begin{array}{l}1.602[0.581- \\
4.420]\end{array}$ & $6 / 8$ & 0.028 & $2.002[1.080-3.711]$ & 1 & 0.009 & \multirow{2}{*}{$\begin{array}{l}\tau^{2}=0.052 \\
\mathrm{QE}(\mathrm{df}=10)=18.03, \mathrm{p} \text {-val } \\
=0.054 \\
\mathrm{~S}^{2}=1.350 \\
\mathrm{I}^{2}=3.737\end{array}$} \\
\hline & Base (milk) & $\begin{array}{l}0.800[0.538- \\
1.191]\end{array}$ & $4 / 4$ & 0.272 & - & & & \\
\hline
\end{tabular}

* N/n Number of studies/number of OR;

** points removed by sensitivity analysis, all results are given after removing data concerned;

B*** Between-study variability $\left(\tau^{2}\right)$, test for residual heterogeneity $(\mathrm{QE})$, variance of residuals $\left(\mathrm{s}^{2}\right)$, intra-class correlation $\left(\mathrm{I}^{2}\right)$.

studies conducted after 2000 produced significantly higher ORs than the older case-control studies did (Table 2). Contact with an ill person or a person having diarrhoea is another significant risk factor, with pooled OR ranging from 2.503 to 5.722 for the three population classes (Table 2 and Figure 2).

\subsubsection{Meta-analysis for food consumption}

Meat, dairy, seafood (only comprising fish), and composite foods were associated with sporadic STEC infection whereas food subcategories like eggs and produce (which included fruits and vegetables) were not. The meat category, which comprised ground beef, undercooked beef, meatballs, ham, salami, hamburgers, hotdogs, donner

\begin{tabular}{|c|c|c|c|c|c|}
\hline Study & Country & Label & Odds Ratio & {$[95 \% \mathrm{Cl}]$} & \\
\hline Kassenborg_CID_2004 & USA & Visit farm with cows & 6.2 & {$[2-19]$} & $\longrightarrow$ \\
\hline Kassenborg_CID_2004* & USA & Visit farm with cows & 10 & {$[1.8-53]$} & $\longrightarrow$ \\
\hline Jaros_BMCID_2013* & NewZealand & Beef in meshblock & 2.61 & {$[1.65-4.13]$} & - \\
\hline Jaros_BMCID_2013* & NewZealand & Dairy in meshblock & 3.26 & {$[1.74-6.13]$} & $\longrightarrow$ \\
\hline Jaros_BMCID_2013* & NewZealand & Living on farm w/animals & 2.74 & [1.74-4.31] & $\longrightarrow-$ \\
\hline Jaros_BMCID_2013* & NewZealand & Animal manure or compost & 2.6 & {$[1.61-4.22]$} & -든 \\
\hline Jaros_BMCID_2013* & NewZealand & Cattle in meshblock & 1.89 & {$[1.04-3.42]$} & $\longrightarrow$ \\
\hline Jaros_BMCID_2013* & NewZealand & Animal manure or compost & 2.09 & {$[1.12-3.9]$} & $\longrightarrow$ \\
\hline Mead_OI_1997 & USA & Visit or live on a farm & 6 & {$[0.6-57.7]$} & \\
\hline OBrien_EID_2001* & UK & Farm contact & 2.45 & {$[1.49-4.02]$} & $-a$ \\
\hline Parry_Lancet_1998 & UK & Living on a farm & 2.9 & {$[0.81-14.42]$} & 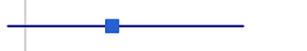 \\
\hline Parry_Lancet_1998 & UK & Visiting private farm & 3 & [0.93-11.23] & \\
\hline Parry_Lancet_1998 & UK & Visiting open farm & 1.36 & {$[0.35-4.7]$} & \\
\hline Parry_Lancet_1998* & UK & Visiting private farm & 7.45 & [1.44-38.4] & \\
\hline Voestsch_Epilnf_2007* & USA & Visiting/living on a farm & 3.2 & {$[1.6-6.1]$} & $\longrightarrow$ \\
\hline Locking_Epilnf_2001 & UK & Visited a farm & 2.34 & {$[1.56-3.69]$} & $\longrightarrow$ \\
\hline Locking_Epilnf_2001 & UK & Ate at farm or zoo & 1.26 & {$[0.39-4.06]$} & 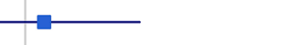 \\
\hline
\end{tabular}

Figure 3. Forest-plot of the association of STEC infection with contact with farm environment in the mixed population (n=25) (* adjusted OR) 


\begin{tabular}{lllccc} 
Study & Country & Label & Odds Ratio & [95\% Cl] \\
\hline Hundy_CDI_2004 & Australia & Chicken & 3.5 & {$[0.45-27.13]$} \\
Hundy_CDI_2004 & Australia & Turkey & 4 & {$[0.36-44.11]$} & \\
McPherson_CID_2009 & Australia & Chicken & 2.2 & {$[0.5-7.4]$} \\
McPherson_CID_2009 & Australia & Chicken & 2.8 & {$[1-7.5]$} & \\
McPherson_CID_2009* & Australia Sliced processed chicken & 4 & {$[1.3-11.6]$} & \\
OBrien_EID_2001* & UK & Rare chicken & 5.13 & {$[1.44-18.26]$} & \\
Bryant_JID_1989 & Canada & Chicken & 3.13 & {$[0.31-31.19]$} & \\
\hline Random Effect Meta-Analysis & All & & 3.55 & {$[1.99-6.33]$} & \\
\hline
\end{tabular}

Figure 4. Forest plot of the association of STEC infection with consumption of poultry in the mixed population ( $\mathrm{n}=7$ ) (* adjusted OR)

kebab and poultry, was significantly associated with STEC infection in all population classes, with pooled ORs ranging from 1.688 to 1.888 (Table 2). Dairy products, which included raw milk, ice cream prepared on farm, formula milk and cream cheese, were significant when considering the whole "children" class (pooled OR=2.240; 95\% CI: 1.161-4.320, Table 2). Composite foods were associated with STEC infection in both the mixed and child populations, with pooled ORs ranging from 1.587 to 3.556 (Table 2). The consumption of fish was significantly associated with STEC infection in the mixed population (pooled OR=1.720; 95\% CI: 1.100-2.687, Table 2).

Within meats, a significant association in the mixed and child populations was found for consumption of beef $(\mathrm{OR}=1.877$; $95 \% \mathrm{CI}$ : 1.513-2.329, Table 3); processed meats, i.e. cold/deli meats, ham, sausage rolls, salami, corned beef, bacon, raw spreadable sausage, precooked meat and burger ( $\mathrm{OR}=1.513$; 95\% CI: $1.227-1.866$, Table 3); and "other meats" encompassing any meat with no specific origin, barbecued meat, donner kebab meat and meat casseroles (pooled OR ranging from 1.803 to 2.574 , Table 3). Interestingly, poultry meat (comprising mostly chicken) presented a higher association with STEC disease than beef/other meats at a pooled OR of 3.549 (95\% CI: 1.990-6.330, Table 3 and Figure 4); although it should be kept in mind that only 7 ORs were available.

Meat-based ready-to-eat (RTE) food, including salami, tartare, deli meats, hotdog, ham and processed chicken (pooled OR from 1.387 to 1.951, Table 3) and barbecued meats (pooled OR $=1.516$; $95 \% \mathrm{CI}$ : $1.000-2.298$, Table 3 ) were also significant risk factors. The strongest associations with beef were found for the mixed population (pooled $\mathrm{OR}=1.904 ; 95 \% \mathrm{CI}: 1.462-2.479$ ) and older children (pooled $\mathrm{OR}=2.216$; 95\% CI: 1.399-3.509, Table 3) compared to the young children (pooled $\mathrm{OR}=1.200$; 95\% CI: 0.885-1.625, Supplementary Material 2). This may arise from the lower exposure of young children to undercooked meats. However, young children were more likely to acquire STEC infection by consuming processed meats (i.e., raw spreadable sausage and minced meat sausages; pooled OR=1.830; 95\% CI: $1.093-3.065$, Table 3) than older children.

Within dairy products, the consumption of raw milk was a significant risk factor for children (both age groups; pooled $\mathrm{OR}=4.117,95 \% \mathrm{CI}$ : 1.043-16.255). The strongest association was found for young children (pooled OR=7.188; 95\% CI: 2.387-21.643, Table 3 and Figure 5)

\subsubsection{Meta-analysis on the effects of handling and preparation of foods}

For some food classes, for which relevant information was available, the effects of handling and setting were appraised (Table 4). Metaanalytical models were adjusted to data partitions suitable for this analysis: (i) beef, other (non-classifiable) meats and minced meat; (ii) processed meat; and (iii) milk. Consuming beef prepared outside the home did not come up as a significant factor for sporadic STEC infection as the odds of infection only marginally increased by 1.106 (95\% CI: $0.895-1.368$, Table 4) in comparison to the base scenario. However, eating undercooked beef/minced beef/other meats was generally a risky practice, as it significantly increased the odds of acquiring STEC infection by 1.470 (95\% CI: $1.217-1.766$, Table 4). Drinking unpasteurized milk caused a two-fold increase (95\% CI: 1.080 - 3.709, Table 4) in the odds of acquiring a sporadic STEC infection.

For most of the meta-analytical models reported in tables 2 and 3, the statistical tests indicated the absence of potential significant publication bias ( $\mathrm{p}>0.05)$. Exceptions were observed in data partitions related to travel (Table 2), food in the mixed population (Table 2), meat in the mixed population (Table 3), raw milk in children (Table 3) and composite foods in the mixed population (Table 3). The funnel plots for these data partitions (Figure 6) suggest that small studies associated with nonsignificant results (i.e., ORs not showing significant associations between food consumption and disease) may have remained unpublished. Intra-class correlation $\left(\mathrm{I}^{2}\right)$ appeared as low $(<25 \%)$ to moderate $(50 \%$ or

\begin{tabular}{|c|c|c|c|c|c|c|}
\hline Study & Country & Label & Odds Ratio & {$[95 \% \mathrm{Cl}]$} & & \\
\hline Belongia_JID_2003 & USA & Unpasteurised milk & 0.85 & {$[0.47-1.53]$} & - & \\
\hline Bielaszewska_Epilnf_1997 & CzechRep & Raw goat's milk & 49.5 & {$[2.53-\operatorname{Inf}]$} & & \\
\hline Rowe_Epilnf_1993 & Canada & Unpasteurised milk & 9.77 & {$[0.98-97.36]$} & & $\longrightarrow$ \\
\hline Werber_AJE_2007 & Germany & Raw milk & 4.5 & {$[1-20.8]$} & & \\
\hline Werber_AJE_2007* & Germany & Raw milk & 6.9 & {$[1-47.9]$} & & $\rightarrow$ \\
\hline Random Effect Meta-Analysis & All & & 4.12 & {$[1.04-16.26]$} & & - \\
\hline
\end{tabular}

Figure 5. Forest-plot of the association of STEC infection with consumption of raw milk in children ( $=5$ ) (* adjusted OR) 

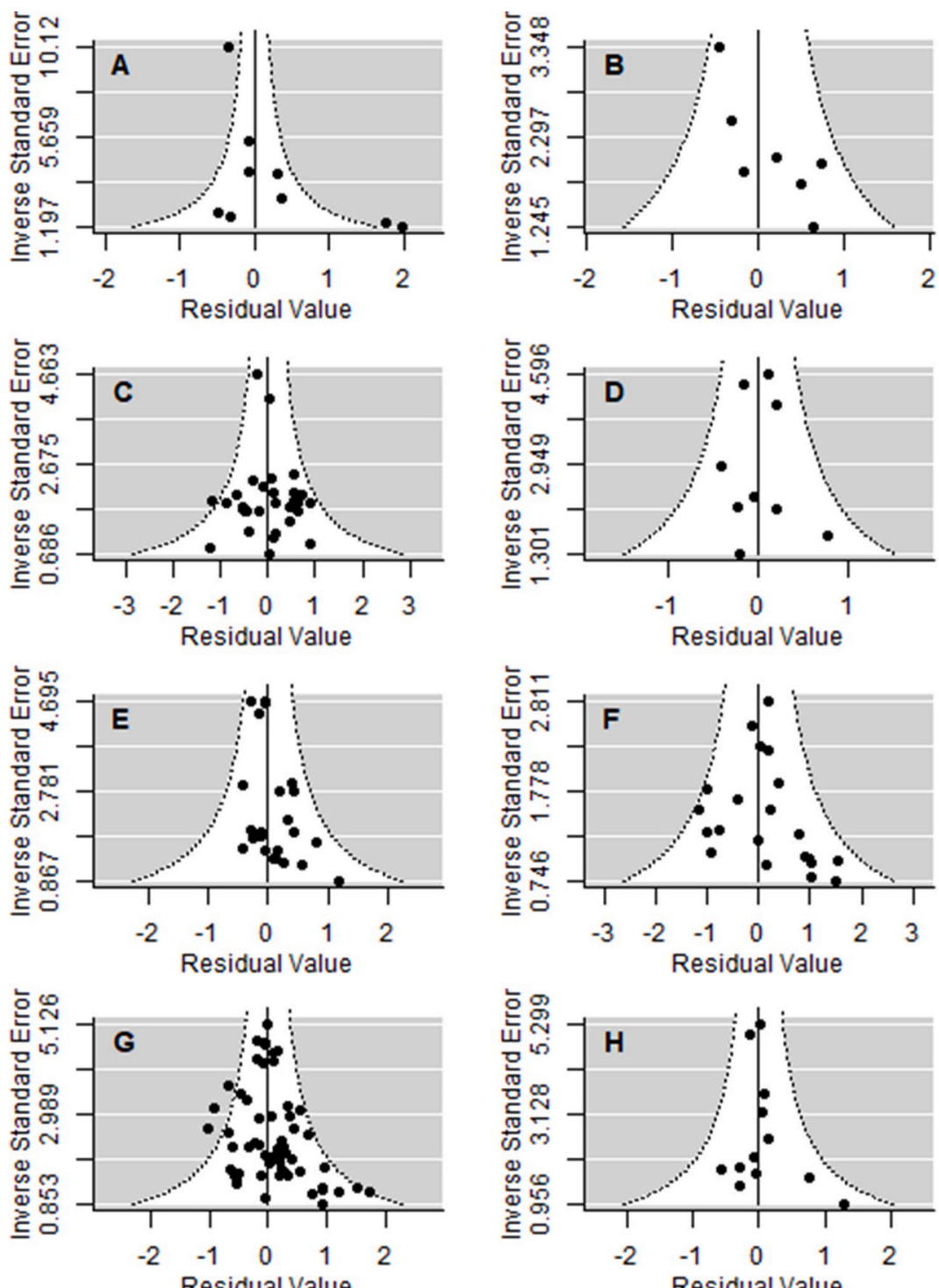

Residual Value
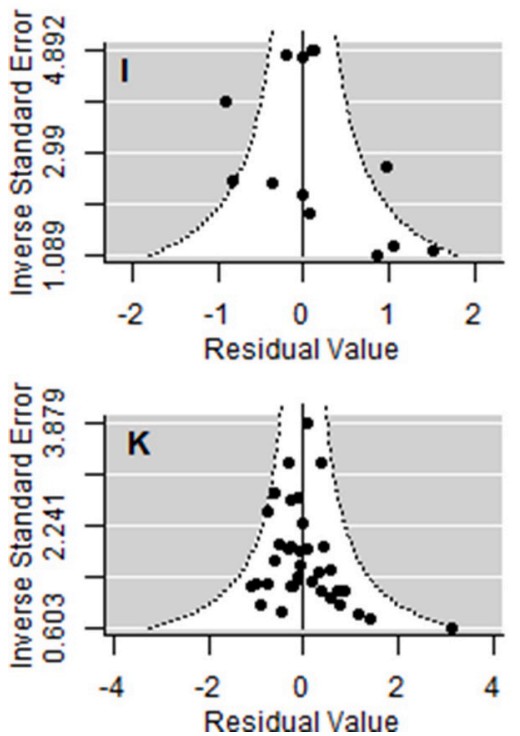

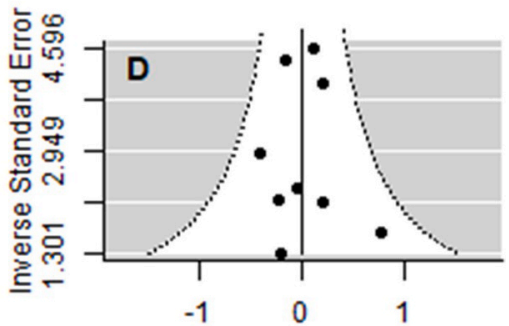

Residual Value

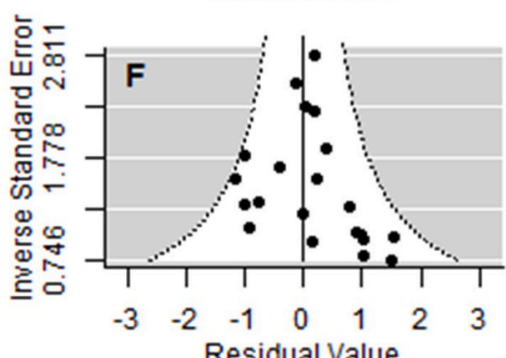

Residual Value
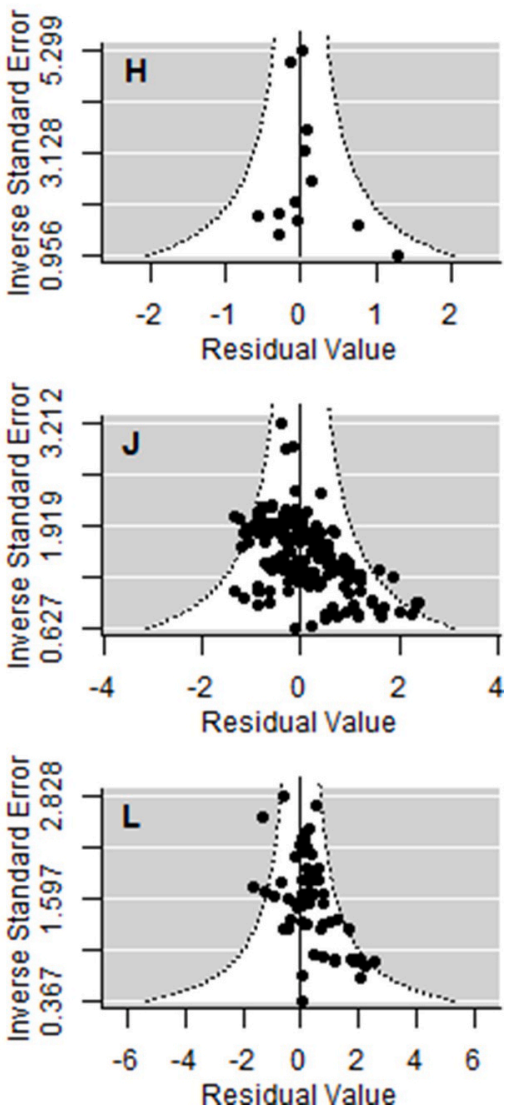

Figure 6. Funnel-plots from meta-analyses investigating categorized risk factors for transmission of STEC infection A: Travel in mixed population, B: Host-specific in mixed population, C: Animals in mixed population, D: Animals in young children, E: Animals in older children, F: Person-to-person in mixed population, G: Environment in mixed population, H: Environment in old children, I: Environment in young children, J: Food in mixed population, K: Food in young children, L: Food in older children. 
around 50\%), while residual between-study heterogeneity was observed in most data partitions (Tables 2, 3 and 4).

\section{Discussion}

This meta-analysis showed that travelling, person-to person transmission; contact with farm animals or their environment, environmental (untreated drinking or recreational water) and food exposures are the main risk factors for sporadic STEC infection. The results are in accordance with the meta-analysis of Kintz et al. (2017) who found that undercooked meat, animal contact and person-to-person transmission were the most frequent routes of transmission of the infection.

In the mixed population, pooled ORs related to exposure to ill people, the farm environment, untreated drinking or recreational water, and farm animals ranged from 1.5 to 3.0. For the child populations, the pooled ORs increased to 4.4 and 5.3 for contact with farm animals and the farm environment, respectively, and to 5.7 for contact with ill persons. Contact with ill persons was a major risk factor for adults and children. This finding is in line with the high rate of secondary transmission observed during STEC outbreaks, particularly at home or among young children in communities (Garvey et al., 2016; Locking et al., 2011; Snedeker et al., 2009).

Direct and indirect contact with farm animals was a risk factor for the mixed population and children (Figure 3 ) which is supported by previous studies. Indeed, several ecological studies showed that living in areas with high densities of farm animals (cattle) was associated with increased risk of sporadic 0157 infection, in particular for young children (Bifolchi et al., 2014; Elson et al., 2018; Friesema et al., 2011; Haus-Cheymol et al., 2006). Furthermore, outbreaks associated with animal contact and environmental exposures (recreational and drinking water) have been also reported (Conrad et al., 2017; Saxena et al., 2015).

The ORs assigned to main food categories ranged from 1.6 to 1.8 for the mixed populations and from 1.7 to 3.6 for child populations. When specific foods at risk are targeted, such as beef or processed meats, the pooled ORs were slightly higher. The highest OR was observed for raw milk products in the young child population with a value of 7.188.

The meta-analysis identified meat and especially beef, but also meat with no specific origin, barbecued meat, donner kebab meat and meat casseroles - mainly when they are consumed undercooked - processed meats and RTE meats as the main risk factors for STEC infection. Raw milk was also identified for the child population. Most of these foods have already been identified as risk factors for sporadic STEC infection (Devleesschauwer et al., 2019; Hoffmann et al., 2017; Kintz et al., 2017) or as sources of STEC outbreaks (Greig and Ravel, 2009; Pires et al., 2019). Based on a comparative risk assessment approach, Kosmider et al. (2010) also estimated that beef products particularly beef burgers pose the highest risk of STEC O157 human infection compared to lamb and pork in the UK. Older children were more prone to acquire STEC infection through consumption of beef than the young ones. This may arise from the lower exposure of young children to undercooked meats.

Composite foods were also identified with high ORs (1.587 for mixed population and 3.556 for child populations). The importance of this food category mainly consumed outside the home is difficult to grasp because it is extremely heterogeneous in terms of recipe, preparation and consumption behaviour. Poultry meat consumption was also identified as risk factor for sporadic STEC infection. This is surprising because poultry is not recognised as an important source of STEC infection. Yet, this observation is consistent among four case-control studies performed in Australia (2), Canada (1) and United-Kingdom (1). The ORs for consumption of poultry extracted from these studies were quite high, ranging from 2.20 to 5.13 and are shown in a forest-plot in Fig. 4 . Chicken was also identified by Devleesschauwer et al. (2019) but specifically in the Western Pacific region. Chicken was also mentioned as a source only in very few outbreaks in America and Europe (Pires et al., 2019).
The consumption of fish was recognised as being significantly associated with sporadic STEC infections. This was not evidenced by Kintz et al. (2017) nor Devleesschauwer et al. (2019). Moreover, very limited data were available for this food subcategory in our study (5 ORs from 3 studies), so the significance of fish as potential vehicle of transmission of STEC must be considered cautiously. Produce (fruits and vegetables) was not significantly associated with sporadic STEC infection. This observation was confirmed by Devleesschauwer et al. (2019) who also realized a protective effect associated with produce, whereas produce is also one of the three main sources for STEC outbreaks next to beef and dairy (Pires et al., 2019).

Overall, our results are comparable with the meta-analyses conducted by Kintz et al. (2017) and Devleesschauwer et al. (2019). However, some differences can be observed, such as untreated drinking water and raw milk (for children), which were significant risk factors in our study. These differences may be related to the analytical strategy implemented in the present study, as the previous meta-analyses were conducted only on a selection of risk factors with a different statistical approach.

\section{Conclusions}

This meta-analysis confirmed the importance of known risk factors for STEC infection, such as person-to-person contact, contact with farm animals or the farm environment. Regarding foodborne transmission, the consumption of meat and composite foods appeared as risk factors in all studied populations, and raw dairy for children. In particular, consuming beef, processed meat and RTE meats were associated with increased sporadic STEC infection risk, especially when consumed undercooked. Except vegetables, the identified vehicles are all consistent with described outbreaks.

Future case-control studies should be enhanced by broadening the range of foods explored to go beyond the 'usual suspects' (e.g. beef, raw milk, untreated water, etc.) and further explore, for instance, the association with chicken or composite foods. For the latter category, compositional characteristics and preparation methods should also be taken into account. In general, there is a need to systematically identify practices related to different food preparation and consumption habits as to avoid confusion bias. For instance, for dairy products, it is recommended to clearly differentiate between raw/pasteurized milk, soft/ hard/blue veined cheese, etc., as they are likely to entail different levels of risk.

\section{CRediT authorship contribution statement}

Jean-Christophe Augustin: Writing - original draft. Pauline Kooh: Methodology, Project administration, Writing - original draft. Lapo Mughini-Gras: Writing - review \& editing. Laurent Guillier: Writing review \& editing. Anne Thébault: Methodology, Formal analysis, Visualization. Frédérique Audiat-Perrin: Writing - review \& editing. Vasco Cadavez: Methodology, Investigation, Formal analysis. Ursula Gonzales-Barron: Methodology, Investigation, Formal analysis, Writing - review \& editing. Moez Sanaa: Conceptualization, Supervision, Writing - review \& editing.

\section{Declarations of competing interest}

The authors declare no conflict of interest.

\section{Acknowledgments}

The authors would like to thank Anses staff and the members of the Anses Working Group on Source Attribution of Foodborne Diseases: Laurence Watier, Frédéric Carlin, Julie David, Philippe Fravalo, Nathalie Jourdan-Da Silva, Alexandre Leclercq, Nicole Pavio, Isabelle Villena, Nathalie Arnich. The authors are grateful to the Foundation for Science 
and Technology (FCT, Portugal) for financial support through national funds FCT/MCTES to CIMO (UIDB/00690/2020); U. Gonzales-Barron acknowledges the national funding by FCT, P.I., through the Institutional Scientific Employment Program contract.

\section{Supplementary materials}

Supplementary material associated with this article can be found, in the online version, at doi:10.1016/j.mran.2020.100117.

\section{Appendices}

\section{Supplementary material \\ Appendix 1: References of the 29 primary studies \\ Appendix 2: Non-significant results on the main risk factors}

\section{References}

Bifolchi, N., Michel, P., Talbot, J., Svenson, L, Simmonds, K., Checkley, S., Chui, L. Dick, P., Wilson, J.B., 2014. Weather and livestock risk factors for Escherichia coli O157 human infection in Alberta, Canada. Epidemiol. Infect. 142, 2302-2313.

Bruyand, M., Mariani-Kurkdjian, P., Le Hello, S., King, L.-A., Van Cauteren, D. Lefevre, S., Gouali, M., Jourdan-da Silva, N., Mailles, A., Donguy, M.-P., Loukiadis, E., Sergentet-Thevenot, D., Loirat, C., Bonacorsi, S., Weill, F.-X., De Valk, H., Réseau Français Hospitalier de Surveillance du SHU Pédiatrique, 2019. Paediatric haemolytic uraemic syndrome related to Shiga toxin-producing Escherichia coli, an overview of 10 years of surveillance in France, 2007 to 2016. Eurosurveillance 24, 1800068.

Bryant, H.E., Athar, M.A., Pai, C.H., 1989. Risk-factors for Escherichia-coli O157-H7 infection in an urban-community. J. Infect. Dis. 160, 858-864.

Byrne, K., Smith, R, L., Vanstone, G.L., Perry, N.T., Launders, N., Adak, G.K., Godbole, G., Grant, Jenkins, C., 2014. Epidemiology and microbiology of Shiga toxin-producing Escherichia coli other than serogroup 0157 in England, 2009-2013. J. Med. Microbiol 63, 1181-1188.

Cieslak, P.R., Noble, S.J., Maxson, D.J., Empey, L.C., Ravenholt, O., Legarza, G., Tuttle, J., Doyle, M.P., Barrett, T.J., Wells, J.G., McNamara, A.M., Griffin, P.M., 1997. Hamburger-associated Escherichia coli O157:H7 infection in Las Vegas: A hidden epidemic. Am. J. Public Health 87, 176-180.

Conrad, C.C., Stanford, K., Narvaez-Bravo, C., Callaway, T., McAllister, T., 2017. Farm Fairs and Petting Zoos: A review of animal contact as a source of zoonotic Enteric Disease. Foodborne Pathog. Dis. 14, 59-73.

Devleesschauwer, B., Pires, S.M., Young, I., Gill, A., Majowicz, S.E., 2019. Associating sporadic, foodborne illness caused by Shiga toxin-producing Escherichia coli with specific foods: a systematic review and meta-analysis of case-control studies. Epidemiol Infect 147, e235.

EFSA, ECDC, 2018. The European Union summary report on trends and sources of zoonoses, zoonotic agents and food-borne outbreaks in 2017. EFSA Journal 16, $5500,5262$.

EFSA BIOHAZ Panel, E.P.o.B.H., 2013. Scientific Opinion on VTEC-seropathotype and scientific criteria regarding pathogenicity assessment. EFSA Journal 11, 3138.

Elson, R., Grace, K., Vivancos, R., Jenkins, C., Adak, G.K., O’Brien, S.J., Lake, I.R., 2018. A spatial and temporal analysis of risk factors associated with sporadic Shiga toxinproducing Escherichia coli 0157 infection in England between 2009 and 2015. Epidemiol Infect 146, 1928-1939.

Friesema, I.H., Van De Kassteele, J., CM, D.E.J., Heuvelink, A.E., Van Pelt, W., 2011 Geographical association between livestock density and human Shiga toxinproducing Escherichia coli 0157 infections. Epidemiol Infect 139, 1081-1087.

Friesema, I.H.M., Schotsborg, M., Heck, M.E.O.C., Van Pelt, W., 2015. Risk factors for sporadic Shiga toxin-producing Escherichia coli 0157 and non-O157 illness in the Netherlands, 2008-2012, using periodically surveyed controls. Epidemiol. Infect. $143,1360-1367$.

Garvey, P., Carroll, A., McNamara, E., McKeown, P.J., 2016. Verotoxigenic Escherichia coli transmission in Ireland: a review of notified outbreaks, 2004-2012. Epidemiol. Infect 144, 917-926.

Gianviti, A., Rosmini, F., Caprioli, A., Corona, R., Matteucci, M.C., Principato, F., Luzzi, I., Rizzoni, G., 1994. Hemolytic-uremic syndrome in childhood - Surveillance and case-control studies in Italy. Pediatr. Nephrol. 8, 705-709.

Gonzales-Barron, U., Thébault, A., Kooh, P., Watier, L., Sanaa, M., Cadavez, V., 2019. Strategy for systematic review of observational studies and meta-analysis modelling of risk factors for sporadic foodborne diseases. Microbial Risk Analysis, 100082.

Greig, J.D., Ravel, A., 2009. Analysis of foodborne outbreak data reported internationally for source attribution. Int. J. Food Microbiol. 130, 77-87.

Gyles, C.L., 2007. Shiga toxin-producing Escherichia coli: An overview1. J. Anim. Sci. 85, E45-E62.
Haus-Cheymol, R., Espie, E., Che, D., Vaillant, V., H, D.E.V., Desenclos, J.C., 2006. Association between indicators of cattle density and incidence of paediatric haemolytic-uraemic syndrome (HUS) in children under 15 years of age in France between 1996 and 2001: an ecological study. Epidemiol Infect 134, 712-718.

Hoffmann, S., Devleesschauwer, B., Aspinall, W., Cooke, R., Corrigan, T., Havelaar, A. Angulo, F., Gibb, H., Kirk, M., Lake, R., Speybroeck, N., Torgerson, P., Hald, T. 2017. Attribution of global foodborne disease to specific foods: Findings from a World Health Organization structured expert elicitation. PLoS One 12, e0183641.

Karch, H., Bielaszewska, M., Bitzan, M., Schmidt, H., 1999. Epidemiology and diagnosis of Shiga toxin-producing Escherichia coli infections. Diagn. Microbiol. Infect. Dis. 34, 229-243.

Kassenborg, H.D., Hedberg, C.W., Hoekstra, M., Evans, M.C., Chin, A.E., Marcus, R. Vugia, D.J., Smith, K., Ahuja, S.D., Slutsker, L., Griffin, P.M., Emerging Infections Program FoodNet Working Group, 2004. Farm visits and undercooked hamburgers as major risk factors for sporadic Escherichia coli 0157: H7 infection: Data from a case-control study in 5 FoodNet sites. Clin. Infect. Dis. 38, S271-S278.

Kintz, E., Brainard, J., Hooper, L., Hunter, P., 2017. Transmission pathways for sporadic Shiga-toxin producing E. coli infections: A systematic review and meta-analysis. Int. J. Hyg. Environ. Health 220, 57-67.

Kirk, M.D., Pires, S.M., Black, R.E., Caipo, M., Crump, J.A., Devleesschauwer, B., Döpfer, D., Fazil, A., Fischer-Walker, C.L., Hald, T., Hall, A.J., Keddy, K.H., Lake, R. J., Lanata, C.F., Torgerson, P.R., Havelaar, A.H., Angulo, F.J., 2015. World Health Organization Estimates of the Global and Regional Disease Burden of 22 Foodborne Bacterial, Protozoal, and Viral Diseases, 2010: A Data Synthesis. PLoS Med 12, e1001921.

Kosmider, R.D., Nally, P., Simons, R.R.L., Brouwer, A., Cheung, S., Snary, E.L., Wooldridge, M., 2010. Attribution of human VTEC 0157 infection from meat products: A quantitative risk assessment approach. Risk Anal 30, 753-765.

Locking, M.E., O’Brien, S.J., Reilly, W.J., Wright, E.M., Campbell, D.M., Coia, J.E. Browning, L.M., Ramsay, C.N., 2001. Risk factors for sporadic cases of Escherichia coli $\mathrm{O} 157$ infection: The importance of contact with animal excreta. Epidemiol. Infect 127, 215-220.

Locking, M.E., Pollock, K.G., Allison, L.J., Rae, L., Hanson, M.F., Cowden, J.M., 2011. Escherichia coli O157 infection and secondary spread, Scotland, 1999-2008. Emerg Infect Dis 17, 524-527.

Mughini-Gras, L., van Pelt, W., van der Voort, M., Heck, M., Friesema, I., Franz, E., 2018. Attribution of human infections with Shiga toxin-producing Escherichia coli (STEC) to livestock sources and identification of source-specific risk factors, The Netherlands (2010-2014). Zoonoses Public Health 65, e8-e22.

Persad, A.K., LeJeune, J.T., 2014. Animal Reservoirs of Shiga Toxin-Producing Escherichia coli. Microbiol Spectr 2. Ehec-0027-2014.

Piérard, D., Crowcroft, N., De Bock, S., Potters, D., Crabbe, G., Van Loock, F., Lauwers, S., 1999. A case-control study of sporadic infection with 0157 and non-O157 verocytotoxin-producing Escherichia coli. Epidemiol. Infect. 122, 359-365.

Pires, S.M., Evers, E.G., Van Pelt, W., Ayers, T., Scallan, E., Angulo, F.J., Havelaar, A., Hald, T., Schroeter, A., Brisabois, A., Thebault, A., Käsbohrer, A., Schroeder, C., Frank, C., Guo, C., Wong, D.L.F., Döpfer, D., Snary, E., Nichols, G., Spitznagel, H., Wahlström, H., David, J., Pancer, K., Stark, K., Forshell, L.P., Nally, P., Sanders, P., Hiller, P., 2009. Attributing the human disease burden of foodborne infections to specific sources. Foodborne Pathog. Dis. 6, 417-424.

Pires, S.M., Majowicz, S., Gill, A., Devleesschauwer, B., 2019. Global and regional source attribution of Shiga toxin-producing Escherichia coli infections using analysis of outbreak surveillance data. Epidemiol Infect 147, e236.

Rivero, M.A., Passucci, J.A., Rodriguez, E.M., Signorini, M.L., Tarabla, H.D., Parma, A.E., 2011. Factors associated with sporadic verotoxigenic Escherichia coli infection in children with diarrhea from the central eastern area of argentina. Foodborne Pathog. Dis 8, 901-906.

Saxena, T., Kaushik, P., Krishna Mohan, M., 2015. Prevalence of E. coli 0157:H7 in water sources: an overview on associated diseases, outbreaks and detection methods. Diagn. Microbiol. Infect. Dis. 82, 249-264.

Snedeker, K.G., Shaw, D.J., Locking, M.E., Prescott, R.J., 2009. Primary and secondary cases in Escherichia coli 0157 outbreaks: a statistical analysis. BMC Infect. Dis. 9, 144.

Viechtbauer, W., 2010. Conducting Meta-Analyses in R with the metafor Package. 201036, 48.

Voetsch, A.C., Kennedy, M.H., Keene, W.E., Smith, K.E., Rabatsky-Ehr, T., Zansky, S., Thomas, S.M., Mohle-Boetani, J., Sparling, P.H., McGavern, M.B., Mead, P.S., 2007. Risk factors for sporadic Shiga toxin-producing Escherichia coli O157 infections in FoodNet sites, 1999-2000. Epidemiol. Infect. 135, 993-1000.

Wang, X., Taylor, M., Hoang, L., Ekkert, J., Nowakowski, C., Stone, J., Tone, G., Trerise, S., Paccagnella, A., Wong, T., Galanis, E., 2013. Comparison of clinical and epidemiological features of Shiga toxin-producing Escherichia coli $\mathrm{O} 157$ and nonO157 infections in British Columbia, 2009 to 2011. Can. J. Infect. Dis. Med. Microbiol. 24, E102-E106.

Werber, D., Behnke, S.C., Fruth, A., Merle, R., Menzler, S., Glaser, S., Kreienbrock, L., Prager, R., Tschäpe, H., Roggentin, P., Bockemühl, J., Ammon, A., 2007. Shiga toxinproducing Escherichia coli infection in Germany - Different risk factors for different age groups. Am. J. Epidemiol 165, 425-434. 